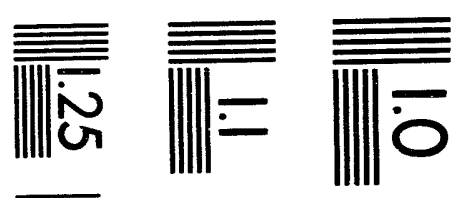

$$
\begin{aligned}
& \text { 可 }
\end{aligned}
$$

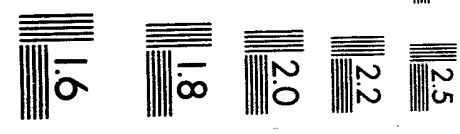



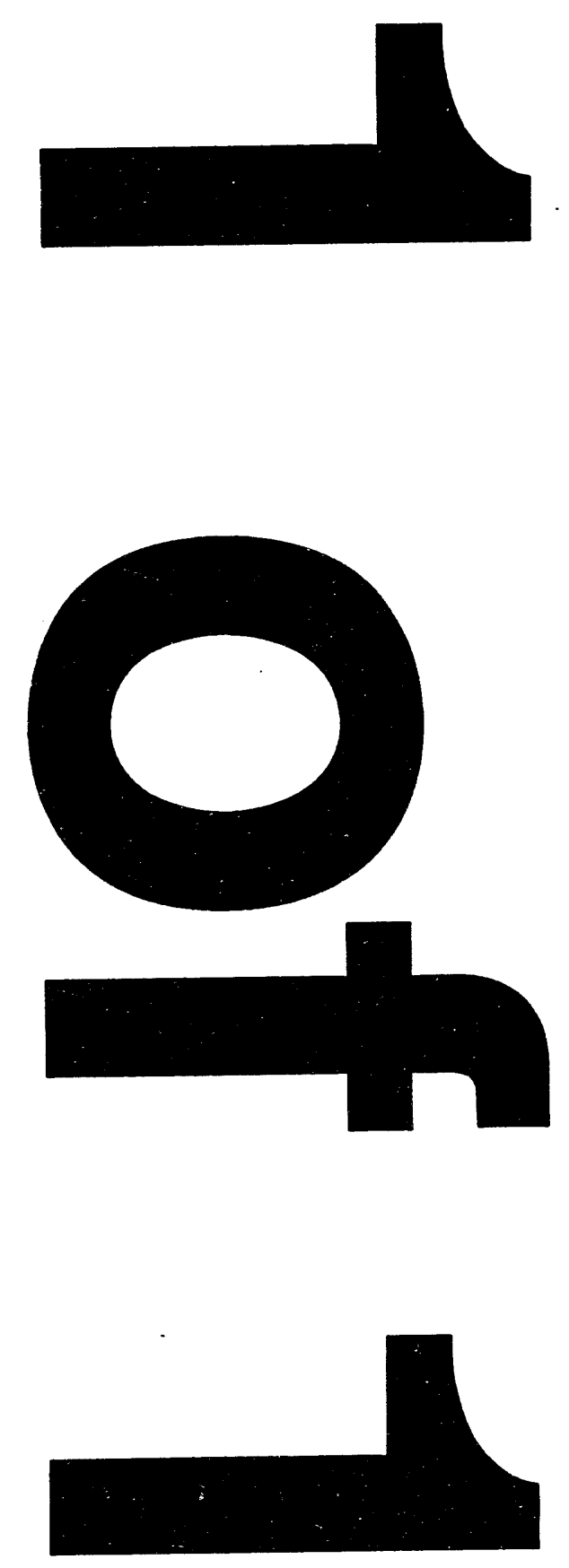


\title{
Using TSAR with the Post-Processing Far-Field Option
}

\author{
Steven T. Pennock
}

\section{October 1993}
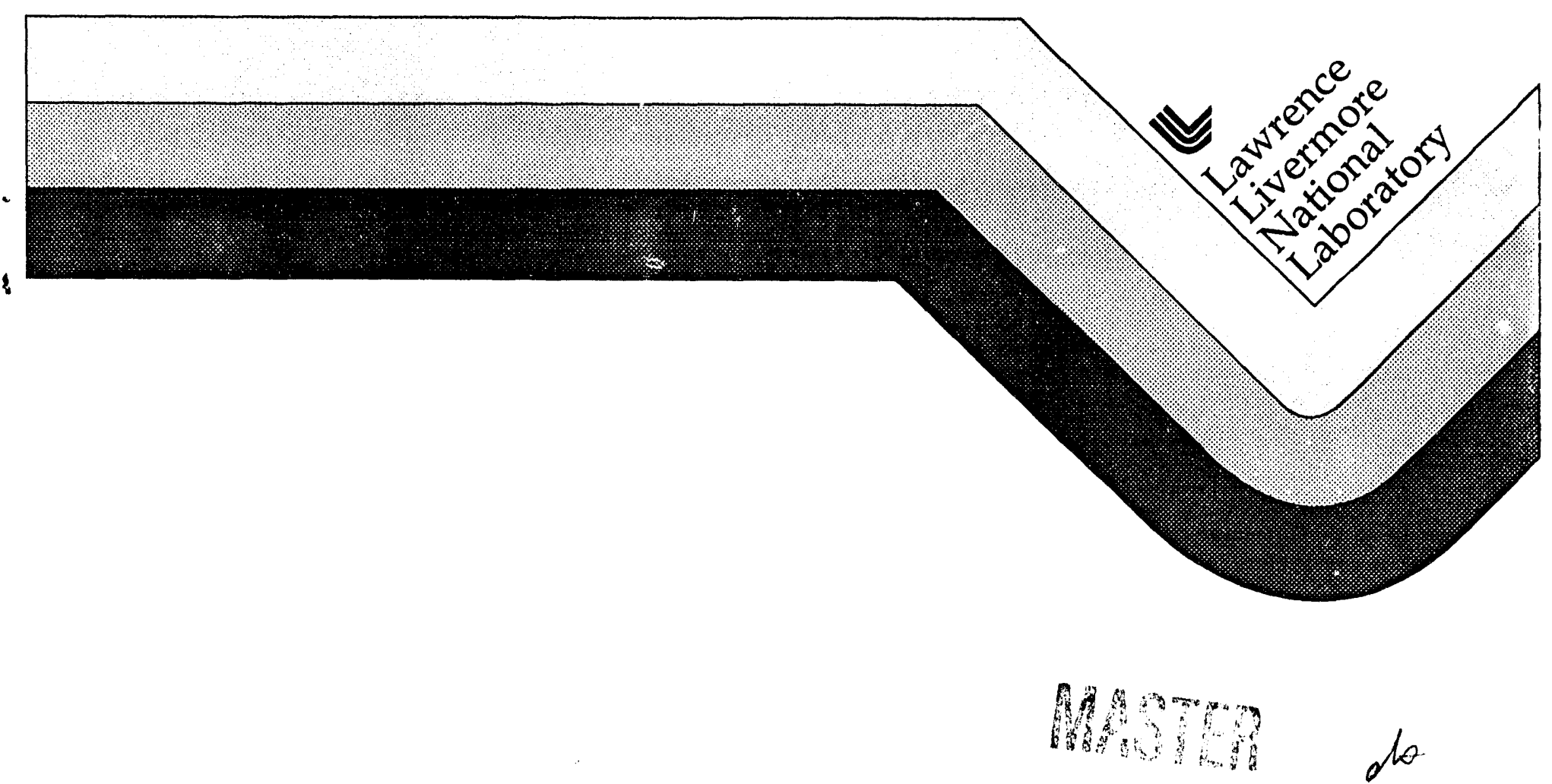


\section{DISCIAINIK}

This document was prepared as an account of work sponsored by an agency of the United States (ioveriment. Neither the United States Government nor the University of California nor any of their employees, makes any warranty, express or implied, or assumes any legal liability or responsibility for the accuracy. completeness. or usefulness of any information, apparatus, product, or process disclosed, or represents that its use would not infringe privately owned rights. Reference herein to any specific commercial products, process, or service by trade name, trademark, manufacturer, or otherwise, does not necessarily constitute or imply its endorsement, recommendation, or favoring by the United States Government or the University of California. The views and opinions of authors expressed herein do not necessarily state or reflect those of the United States Government or the University of (alifornia, and shall not bc used for advertising or product endorsenent purposes.

Work performed under the auspices of the U.S. Department of Energy by Lawrence Livermore National Labor itory under Contract W-7405-Eng-48. 
Using TSAR with the Post-Processing Far-Field Option

Steven T. Pennock

The TSAR EM code has contained far-field projection algorithms for some time. One drawback to previous versions of TSAR has been the necessity to run the field advancement routines each and every time to generate far-field data. A change has been made to TSAR to allow tangential fields on the projection surface to be saved to a file. These fields can then be used with a new postprocessor, POSTFAR, to produce far-field points at any desired location without re-running the field advancement portion of TSAR. This will save large amounts of time in producing far-field calculations and allow calculation of other test point locations. If the tangential field data is saved to an $8 \mathrm{~mm}$ tape, these calculations may be made at a much later date.

To set up a TSAR run to save tangential fields, two lines are added to the tsarin file:

FlagFDmp = .TRUE.

FDmpFile $\quad=$ 'DumpFileName'

The first line simply specifies that the tangential fields are to be saved, while the second line gives the exact file name of the output. This file name can include path information, if the tangential field data will be stored in another directory or on another disk.

The near-field sampling surface needs to be defined in the tsarin file, i.e., the variables NearXMin, NearXMax, etc., need to be specified. This, along with the number of time steps, will also determine the size of the output file. The formula for the file-size is:

$32 \times$ NSteps $x[\{($ NearXMin - NearXMax +1$) \times($ NearYMax - NearYMin +1$)\}+$ $\{($ NearXMax - NearXMin +1) $x($ NearZMax - NearZMin +1$)\}+\{($ NearYMin NearYMax + 1) x (NearZMax - NearYMin +1)\}].

The files generated will be huge and require enormous amounts of disk space. For example, a projection surface of $100 \times 100 \times 100$ cells would require about $1 \mathrm{MB}$ of disk space for each time step. A 2 GB disk would allow approximately 2000 time steps to be stored. Larger sampling surfaces would allow fewer time steps to be saved for a given disk size. It is hoped that disk technology will continue to improve, with resulting larger disk sizes, reducing this limitation.

The tsarin file need not contain any far-field test point location information at run time, although this is not ruled out. TSAR is simply run at this point, the field advancement routines generate the tangential field data and these values are saved in the dump file. Once TSAR finishes, the tangential field data can be used to generate far-field test points at will, using the new code POSTFf?

POSTFAR is very similar to TSAR, making use of the same variable names and input file. Add far-field requests to the tsarin file used to generate the tangential field data, then run POSTFAR. If the tangential field data is located on another disk, a soft link (ln -s filen:me) can be used to simply point to the tsarin file, rather than copying tsarin to the other location. 
One way to avoid creating too many tsarin files is to set up tsarin with everything included, the file dump as well as all the desired far-field locations and orientations. Before running TSAR, edit the file and move all the far-field test point information below the END line. This information will not be read in when TSAR is run. Once the TSAR run finishes, move the far-field information back up above END and run POSTFAR. This assures that all the information for the POSTFAR run matches that for the initial TSAR run.

Since POSTFAR, like TSAR, accumulates all the dipole fields prior to writing any data out, output files will not be opened until the job is nearly finished. A record of the progress and the total time expended will be written to the screen, or to a log file if the job is submitted in the background, (postfar $>\&$ postfar.log \&).

To compile POSTFAR, use the gnumake file GNUmakefile.ppf, which accompanies the latest version of TSAR. The actual command is:

gnumake -f GNUmakefile.ppf postfar

An example tsarin file for use with POSTFAR is shown below. The two lines just before END call for tangential field output, and the lines below END are moved up prior to running POSTFAR.

\begin{tabular}{|c|c|c|}
\hline \multicolumn{3}{|l|}{ \$INPUT } \\
\hline FlagEcho & $=$ & .TRUE., \\
\hline FlagCord & $=$ & .TRUE., \\
\hline FlagExtn & $=$ & .TRUE., \\
\hline ConductE(3) & $=$ & $3.5400 \mathrm{E}+07$ \\
\hline Dx & $=$ & $3.1750 \mathrm{E}-03$ \\
\hline NSteps & $=$ & 1024 \\
\hline OriginI & $=$ & 30.00 \\
\hline OriginJ & $=$ & 25.00 \\
\hline OriginK & $=$ & 25.00 \\
\hline WaveShap & $=$ & 7 \\
\hline PeakTime & $=$ & 80.00 \\
\hline Width & $=$ & 50.00 \\
\hline Peak & $=$ & 1000 \\
\hline Frqncy & $=$ & $7.0000 E+09$ \\
\hline Phi & $=$ & $0.0000 \mathrm{E}+00$ \\
\hline Polar & $=$ & $0.0000 E+00$ \\
\hline Title & $=$ & 'China Lake Box,Gaussian Pulse,H-Pol,0 deg,TFar,7/18/92', \\
\hline GridFile & $=$ & 'cl_box4.grd' \\
\hline ScatXMin & $=$ & 13 \\
\hline ScatXMax & $=$ & 47 \\
\hline ScatYMin & $=$ & 13 \\
\hline ScatYMax & $=$ & 37 \\
\hline ScatZMin & $=$ & 13 \\
\hline ScatZMax & $=$ & 3 \\
\hline BCXMin & $=$ & '2ND ORDER MUR RADIATION' \\
\hline BCXMax & $=$ & '2ND ORDER MUR RADIATION' \\
\hline BCYMin & $=$ & '2ND ORDER MUR RADIATION' \\
\hline BCYMax & $=$ & '2ND ORDER MUR RADIATION' \\
\hline BCZMin & $=$ & '2ND ORDER MUR RADIATION' \\
\hline BCZMax & $=$ & '2ND ORDER MUR RADIATION' \\
\hline
\end{tabular}


$\begin{array}{lll}\text { FlagFDmp } & = & \text { TRUE. } \\ \text { FDmpFile } & = & \text { 'tsarl/pennock/cl_far.dmp' } \\ \text { \$END } & \end{array}$

TFarDir $=2$

TFarR. $=10.00$

TFarThta $=90.00$

TFarPhi $=180.0$

Although this example shows a time-domain far-field test point, any of the standard TSAR projections which depend on equivalent surface currents may be used. These include frequency domain test points and near-field time-domain test points (beyond the computational volume, but closer than the standard far-field distance), as well as timedomain far-field points. 

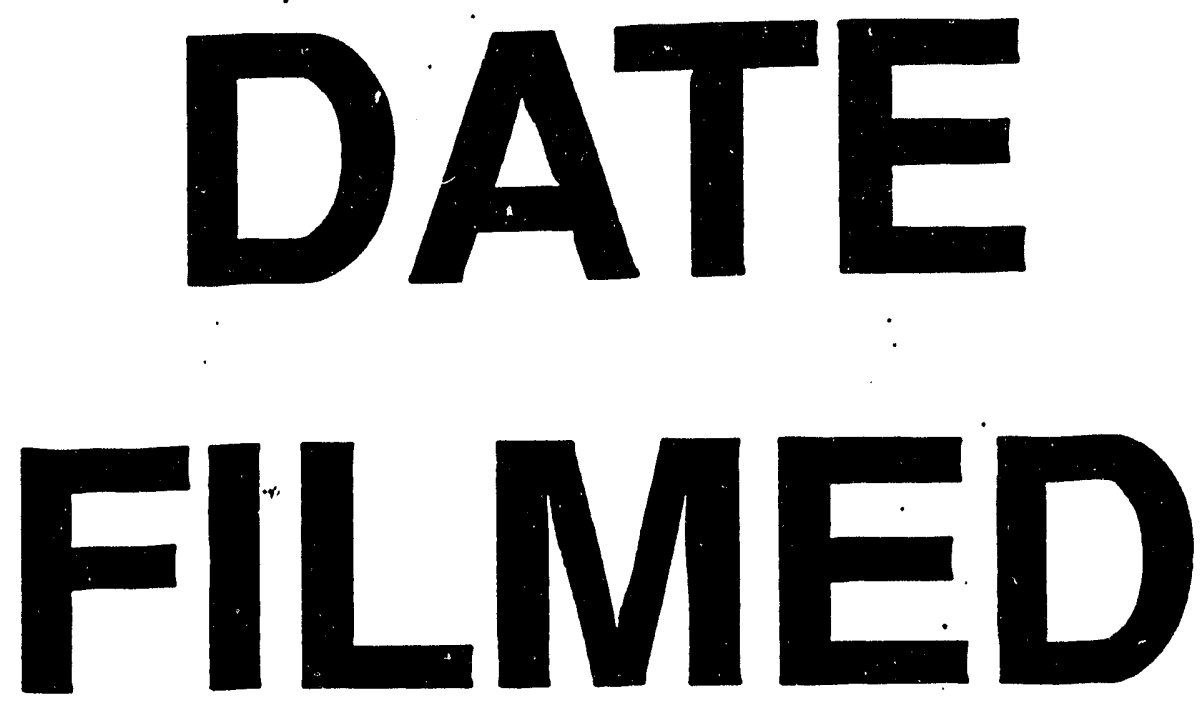

$3 / 22 / 94$
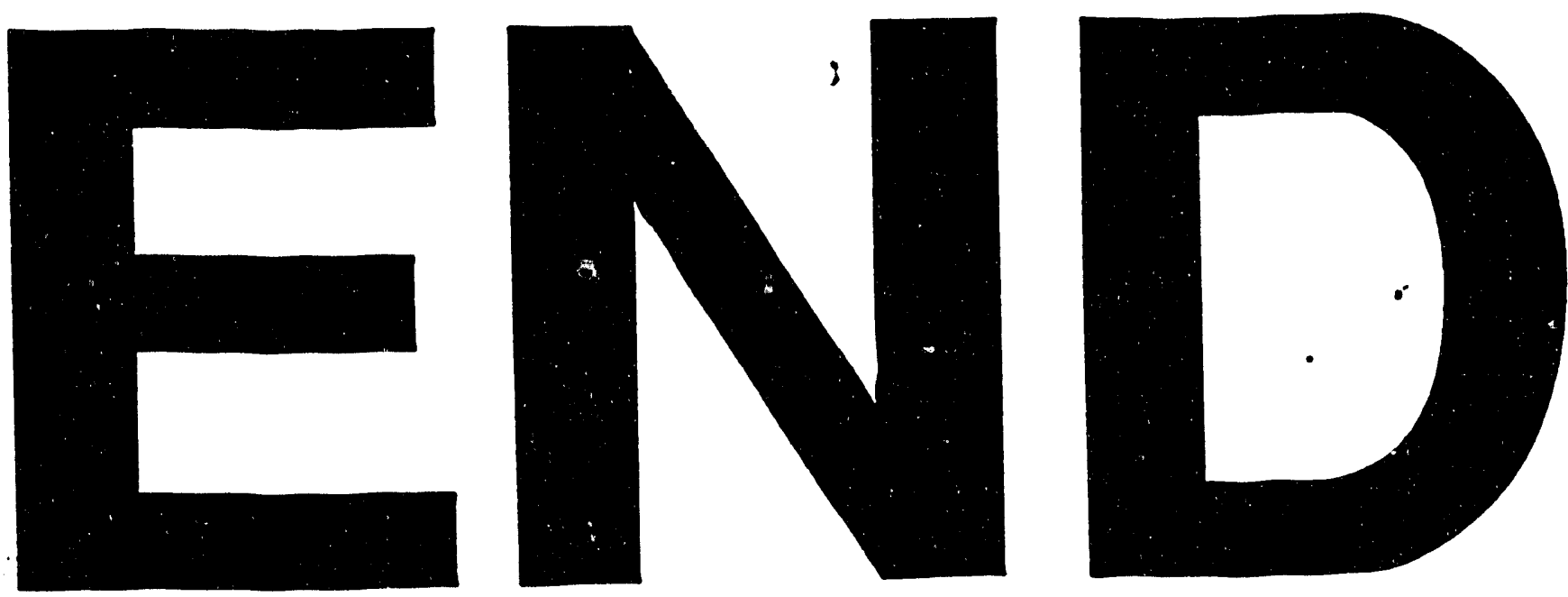
